\title{
Dia Sem Carne: Relato de um Projeto de Extensão
}

\author{
Meatless Day: Report of an Extension Project
}

RESUMO

O projeto de extensão Dia Sem Carne foi elaborado na Faculdade de Saúde Pública da USP, em 2012, com o objetivo de discutir com a comunidade acadêmica sobre os riscos causados à saúde e ao meio ambiente pelo consumo excessivo de carne. Durante o ano de 2012 o projeto foi chamado Segunda Sem Carne na FSP e a ação principal foi a parceria com o restaurante da Faculdade de Saúde Pública da Universidade de São Paulo-COSEAS, no qual houve a inclusão de um cardápio sem carne em uma segunda-feira de cada mês. Além disso, o projeto atingiu o ciberespaço e as mídias sociais, com uma página no Facebook e um blog. Em 2013, o projeto alcançou a comunidade externa à USP, com parceria firmada com a Alimentação Escolar do Município de São Paulo e com o Centro de Referência para prevenção e controle de doenças associadas à Nutrição - CRNutri para a realização de palestras e oficinas para a comunidade, além de continuar com ações digitais por meio do blog e mídia social.

Palavras-chave: Consumo de Carne. Saúde. Ambiente. Nutrição. Relato.

\begin{abstract}
The extension project was developed at School of Public Health (SPH), University of São Paulo, in 2012, and its aims was to discuss with academic people the health and environmental risks caused by excessive meat consumption. In 2012, the project was titled Meatless Monday and it had partnership with the restaurant of the School of Public Health, University of São Paulo-COSEAS, where were offered to population a no-meat menu included on one Monday of each month. Furthermore, a Facebook page and a blog were developed. In 2013, the project reached extramural community with a partnership with the School Feeding Program in São Paulo and the Reference Center on Nutrition and Chronic Diseases Control to develop lectures and workshops for the community. Furthermore, we continued with the blog and social media.
\end{abstract}

\section{Aline Carvalho, Joyce Martins, Camila Negrão, \\ Soraya SElem, Samantha Andrade, Viviane Vieira, Regina Fisberge Dirce Marchioni}

Universidade de São Paulo. Faculdade de Saúde Pública, São Paulo, Brasil 
Keywords: Meat Consumption. Health. Environmental. Nutrition. Report.

\section{INTRODUÇÃO}

A carne tem grande importância na alimentação humana devido à sua composição, com quantidade significativa de proteína, minerais, vitaminas e ácidos graxos [6]. Entretanto, ela vem sendo consumida em quantidades acima das recomendações nacionais e internacionais (uma porção de carne por dia - 19okcal; 500 de carne vermelha e processada por semana, respectivamente) $[6,8]$. Em São Paulo, por exemplo, $75 \%$ das pessoas consomem além do recomendado [1]. O principal problema do consumo excessivo, principalmente das carnes vermelhas e processadas, é a relação com doenças cardiovasculares e câncer, as principais causas de morte no Brasil e no mundo $[4,5]$.

Além do custo à saúde, a produção de carne também gera impacto sobre o meio ambiente, pois promove o desmatamento, efeito estufa, reduz a biodiversidade e afeta a reposição de água doce $[2,7]$.

Pensando nos efeitos que a produção e o consumo de carne causam na saúde e no meio ambiente, foi elaborado em 2012 na Faculdade de Saúde Pública da USP o projeto de extensão. O principal objetivo do projeto foi o de discutir a relação entre o consumo excessivo de carne e o risco à saúde e ao meio ambiente [3].

\section{RELATO}

O projeto de extensão teve dois momentos, o primeiro em 2012, em que era chamado de Segunda Sem Carne na FSP, e o segundo em 2013, no qual houve a mudança de nome para Dia Sem Carne.

\section{Primeiro Momento}

Em 2012, elaborou-se o projeto de extensão Segunda Sem Carne na FSP, e foi usada a identidade visual da ONG Segunda Sem Carne brasileira, após a permissão e aprovação da mesma (Figura 1). 


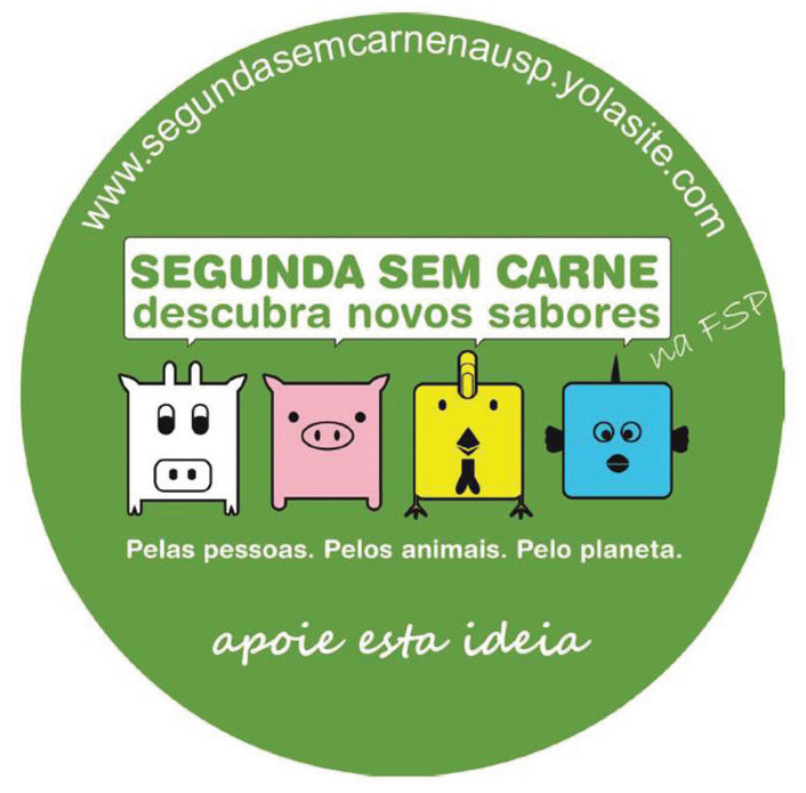

Figura 1 - Logotipo do projeto no primeiro momento.

Nesta fase, o projeto consistiu em intervenções no Restaurante Universitário da Faculdade de Saúde Pública, baseadas em um cardápio sem carne em uma segunda-feira de cada mês do ano.

Foram feitas pesquisas de opinião na saída do restaurante universitário para saber a aceitação das preparações oferecidas e a aceitação do projeto (Figura 2). Mais detalhes podem ser obtidos em outra publicação [3].

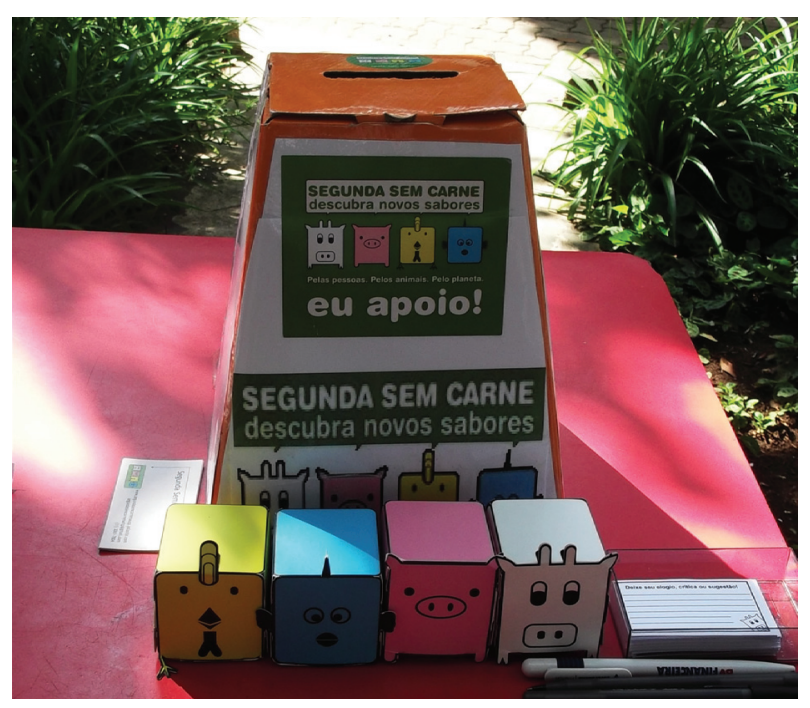

Figura 2 - Urna de votação na saída do restaurante universitário.

Além disso, foi feita extensa divulgação de dados sobre o consumo excessivo de carne e seus impactos na saúde e no meio ambiente por meio de vídeos, site do projeto, 
palestras, boletins informativos, mídias sociais, entrevistas a jornais, revistas, rádios e canais de televisão*. Os materiais divulgados na internet alcançaram usuários de mais de 20 países, como Estados Unidos, França, Reino Unido, Portugal, Argentina, entre outros. No Brasil foram atingidas diversas cidades das regiões Nordeste, Sul, Sudeste e Centro-Oeste e a página do Facebook recebeu cerca de 600 visitas diretas e 4 mil indiretas nesse período. $\mathrm{O}$ site alcançou mais de 2 mil acessos.

\section{Segundo Momento}

No ano de 2013, o projeto mudou de nome e logotipo para desvincular-se da ONG Segunda Sem Carne brasileira, já que os objetivos de ambos não eram totalmente congruentes.

Assim, foi feita uma votação entre os alunos da FSP-USP para uma nova identidade visual (Figura 3 ) e a partir do novo logotipo escolhido, foi criado um novo site e Facebook.

Figura 3 - Logotipo do projeto no segundo momento.

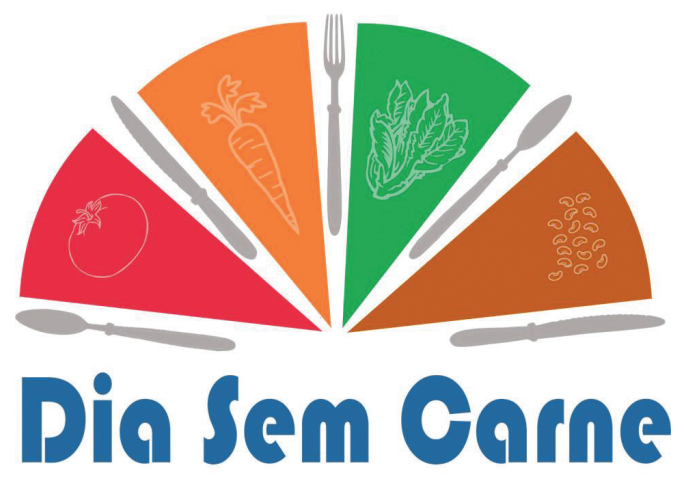

Em 2013, a Superintendência de Assistência Social (SAS-USP) suspendeu para reavaliação todos os projetos que aconteciam nos restaurantes universitários inclusive o Dia Sem Carne. Portanto, não foram feitas intervenções no restaurante universitário da FSP-USP. Entretanto o objetivo do projeto continuou o mesmo: discutir a relação entre o consumo excessivo de carne e o risco à saúde e ao meio ambiente dentro e fora da Universidade. Assim foram feitas as parcerias e atividades listadas abaixo:

1. Parceria com o Departamento de Alimentação Escolar da Prefeitura de São Paulo, a fim de elaborar material didático para crianças, pais e funcionários explicando o projeto que já existe na prefeitura de São Paulo, no qual não é servido carne duas vezes por mês nas escolas da rede básica de ensino do município de São Paulo.

*Disponível em: <www.segundasemcarnedafsp.yolasite.com> 
2. Apresentação de duas palestras para os profissionais do Departamento de Alimentação Escolar, a fim de discutir os impactos na saúde do consumo excessivo de carne.

3. Apresentação de uma palestra sobre consumo excessivo de carne e a experiência do Dia Sem Carne na Universidade Estadual de Campinas no Seminário de Segurança Alimentar e Nutricional.

4. Parceria com o CRNutri, na qual foram feitos uma atividade educativa em comemoração ao Dia do Nutricionista e um grupo temático sobre o consumo excessivo de carne, ambos abertos para comunidade, alunos e funcionários da Faculdade de Saúde Pública da USP.

A atividade educativa teve como tema Está faltando criatividade para montar sua refeição? Aprenda deliciosas receitas para incluir os grãos no seu dia a dia sem cair na monotonia!. Foi feita uma dinâmica com os participantes explicando as recomendações de consumo de carne e como adequar a alimentação com aumento do consumo de grãos.

O grupo temático se deu em três encontros semanais com duração de uma hora e meia cada. Os encontros foram realizados em formato dinâmico para que os participantes pudessem interagir e discutir. Ao final de cada encontro, os participantes recebiam material com o resumo dos conceitos mais importantes (Figura 4).

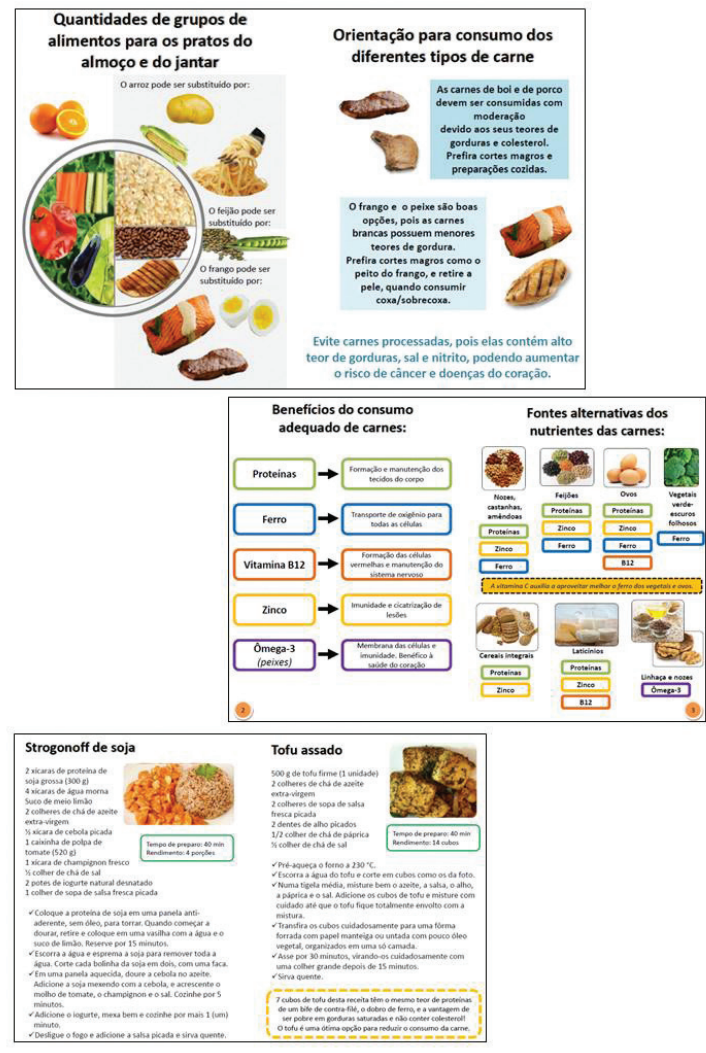

Figura 4 - Materiais entregues ao grupo temático. 
No primeiro encontro foi explorado o tema Benefícios do consumo moderado de carnes, no qual houve a discussão sobre diferença entre carnes frescas (carnes bovinas, suínas, frangos e peixes) e processadas, e quais os nutrientes presentes nas carnes por meio de um jogo de perguntas e respostas (Tabela 1).

Tabela 1 - Jogo de perguntas e respostas utilizadas no grupo temático.

\begin{tabular}{|c|c|c|}
\hline NUTRIENTES & $\begin{array}{l}\text { PERGUNTAS } \\
\text { REALIZADAS }\end{array}$ & $\begin{array}{l}\text { ALIMENTOS } \\
\text { CORRESPONDENTES }\end{array}$ \\
\hline Proteínas & $\begin{array}{l}\text { G1: Sou o principal nutriente da } \\
\text { carne, responsável pela formação } \\
\text { e manutenção dos tecidos do } \\
\text { corpo. Quem sou? } \\
\text { G2: Somos o casal perfeito. } \\
\text { Nascemos no Brasil e não } \\
\text { sabemos viver separados! Quem } \\
\text { é? }\end{array}$ & Arroz + Feijão \\
\hline Ferro & $\begin{array}{l}\text { G1: Sou um mineral importante } \\
\text { para transportar oxigênio para o } \\
\text { corpo e fico dentro das células } \\
\text { vermelhas do sangue. Quem sou? } \\
\text { G2: Sou um alimento de origem } \\
\text { vegetal rico em ferro, mas preciso } \\
\text { de uma mãozinha de frutas } \\
\text { cítricas para que meu ferro seja } \\
\text { bem absorvido. Quem sou? }\end{array}$ & Feijão + Laranja \\
\hline Vitamina B12 & $\begin{array}{l}\text { G1: Sou uma vitamina necessária } \\
\text { para a formação das células } \\
\text { vermelhas do sangue e para a } \\
\text { manutenção do sistema nervoso. } \\
\text { Quem sou? } \\
\text { G2: A vitamina B12 pode ser } \\
\text { encontrada em quais outras } \\
\text { fontes alimentares? }\end{array}$ & Leite + Queijo + Ovos \\
\hline Zinco & $\begin{array}{l}\text { G1: Sou um mineral essencial } \\
\text { para o funcionamento do sistema } \\
\text { imunológico e auxilio em diversas } \\
\text { reações biológicas do organismo. } \\
\text { Quem sou? } \\
\text { G2: Somos um grupo de } \\
\text { alimentos chamado de } \\
\text { oleaginosas, e somos ricas em } \\
\text { zinco. Quem somos? }\end{array}$ & Nozes + Castanhas \\
\hline
\end{tabular}


No segundo encontro, o tema foi Recomendações para o consumo de carne e prejuízos para a saúde e meio ambiente, no qual foi demostrada a quantidade de carne que eles relataram consumirem habitualmente e a quantidade que eles deveriam ter consumido, segundo as recomendações nacional [6] e internacional [8]. Utilizou-se de protótipos para que os participantes visualizassem o tamanho das porções de algumas carnes. Além disso, utilizou-se um vídeo para mostrar o impacto da produção de carne bovina no meio ambiente**.

No último encontro, trabalhou-se o tema Métodos de preparo, cortes magros e substitutos saudáveis para a carne, no qual foram mostrados os tipos de cortes, processamento culinário e os substitutos da carne. Foram desenvolvidas receitas sem carne para os participantes provarem e foi entregue uma cartilha com receitas para incentivar que as novas preparações fossem incorporadas na dieta habitual.

5. A equipe do projeto participou como a única equipe brasileira do prêmio português AUA (Angelini University Award) para práticas de nutrição, ficando entre os finalistas. $\mathrm{O}$ vídeo enviado contou os objetivos do projeto, assim como as atividades desenvolvidas e o que se espera de mudanças no hábito alimentar da população a partir das ações do projeto.

Continuou-se com as ações digitais por meio do blog e do Facebook, porém foram criadas novas versões com o novo logotipo desenvolvido. Foram criados infográficos, receitas e matérias sobre consumo de carne, saúde e meio ambiente. A página do $\mathrm{Fa}$ cebook teve alcance de mais de 14 mil usuários durante o ano, mais de 250 curtidas na página e quase 600 acessos no site**.

Recebeu-se também uma verba da Pró-Reitoria de Cultura e Extensão Universitária da USP para realização, em 2014, de um documentário sobre o projeto e o consumo excessivo de carne, que está em andamento e tem o objetivo de ampliar a discussão sobre o consumo excessivo de carne.

\section{CONCLUSÃO E PRÓXIMOS PASSOS}

O projeto teve importantes ganhos em seus dois momentos, no primeiro fazendo intervenções na Faculdade de Saúde Pública mensalmente e conseguindo sensibilizar aos alunos e funcionários para que mudassem seus hábitos alimentares. No segundo momento, expandindo sua atuação para a comunidade a partir da parceria com o CRNutri e com o Departamento de Alimentação Escolar da Prefeitura de São Paulo, além dos usuários do Facebook, do site e do blog.

Pretende-se manter as atividades atuais das redes sociais e site, que levam informação à população, e a finalização do documentário para ampliação do debate sobre

\footnotetext{
**Disponível em: <http://www.youtube.com/watch?v=HZOm6od2Npl>

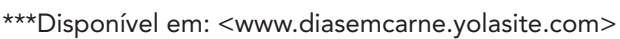


os impactos do consumo excessivo de carne.

\section{REFERÊNCIAS}

[1] CARVALHO, A. M.; et al. Excessive meat consumption in Brazil: Diet quality and environmental impacts. Public Health Nutrition, n. 16, pp. 1893-1899, 2012. DOI:10.1017/S1368980012003916.

[2] CEDEBERG, C.; et al. Including carbon emissions from deforestation in the carbon footprint of Brazilian beef. Environ Sci Technol., n. 45, pp. 1773-1779, 2011.

[3] LACERDA, B.; et al. Segunda Sem Carne na Faculdade de Saúde Pública: Um projeto de intervenção. Revista de Cultura e Extensão USP, Brasil, v. 10, pp. 113-119, nov. 2013. ISSN 2316-906o. Disponível em: <http://www.revistas.usp.br/rce/article/view/69055/71504>. DOI: http://dx.doi.org/10.11606/ issn.2316-906o.v1oiop113-119.

[4] MCAFEE, A. J.; et al. Red meat consumption: An overview of the risks and benefits. Meat Scienc., v. 84, pp. 1-13, 2010.

[5] MICHA, R.; WALLACE, S. K.; MOZAFFARIAN, D. Red and processed meat consumption and risk of incident coronary heart disease, stroke, and diabetes mellitus: A systematic Review and Meta-Analysis. Circulation., v. 121, pp. 22712283, 2010.

[6] MINISTÉRIO DA SAÚDE. Secretaria de Atenção à Saúde. Coordenação-Geral da Política de Alimentação e Nutrição. Guia alimentar para a população brasileira: Promovendo a alimentação saudável. Brasília: Ministério da Saúde, 2006.

[7] STEINFELD. H.; et al. Livestock's long shadow: Environmental issues and options. Food and Agriculture Organization of the United Nations: Roma, 2006.

[8] WORLD CANCER RESEARCH FUND/AMERICAN INSTITUTE FOR CANCER RESEARCH. Food, Nutrition, Physical Activity, and the Prevention of Cancer: A Global Perspective. Washington DC: AICR, 2007.

\section{AGRADECIMENTOS}

Agradecemos a toda equipe da Superintendência de Assistência Social da USP (SAS-USP), nutricionistas e funcionários do Restaurante Universitário da Faculdade de Saúde Pública, alunos de graduação e funcionários do TIC-FSP-USP, aprimorandas e nutricionistas do CRNutri pelo apoio e ajuda para realizar o projeto, ao Departamento de Alimentação Escolar da Prefeitura de São Paulo.

À diretora da FSP-USP, Helena Ribeiro, e ao Departamento de Nutrição da FSP-USP pelo apoio institucional.

Ao Segunda Sem Carne Brasil pelo apoio e discussões. 
ALINE MARTINS DE CARVALHO doutoranda em Nutrição em Saúde Pública da Faculdade de Saúde Pública da Universidade de São Paulo (FSP-USP) - e-mail:alinenutri@usp.br

JOYCE GODIN HO MARTINS graduanda em Nutrição da Faculdade de Saúde Pública da Universidade de São Paulo (FSP-USP) - e-mail: joy.godinho@gmail.com

CAMILA NEGRÃo nutricionista formada pela Faculdade de Saúde Pública da Universidade de São Paulo (FSP-USP) - e-mail: k.nutriusp@gmail.com

SORAYA SANT'ANA DE CASTRO SELEM mestre em Nutrição em Saúde Pública pela Faculdade de Saúde Pública da Universidade de São Paulo (FSP-USP) - e-mail:sorayaselem@gmail.com

SAMANTHA CAESAR DE ANDRADE doutora em Nutrição em Saúde Pública pela Faculdade de Saúde Pública da Universidade de São Paulo (FSP-USP) e nutricionista do Centro de Saúde Escola Geraldo de Paula Souza - e-mail: sami@usp.br

VIVIANE LAUDE LINO VIEIRA doutora em Nutrição em Saúde Pública pela Faculdade de Saúde Pública da Universidade de São Paulo (FSP-USP) e nutricionista do Centro de Saúde Escola Geraldo de Paula Souza-e-mail: vivianevieira@usp.br

RE GINA MARA FISBERG professora do Departamento de Nutrição da Faculdade de Saúde Pública da Universidade de São Paulo (FSP-USP) - e-mail:rfisberg@usp.br

DIRCE MARIA MARCHIONI professora do Departamento de Nutrição da Faculdade de Saúde Pública da Universidade de São Paulo (FSP-USP) - e-mail: marchioni@usp.br 